\title{
Análise do Tempo de Duração de Rotas em Redes Veiculares Considerando Fatores Influentes
}

\author{
Danilo R. de Assis ${ }^{1}$, Joilson Alves Junior ${ }^{1}$, Emilio C. G. Wille ${ }^{1}$ \\ ${ }^{1}$ Universidade Tecnológica Federal do Paraná (UTFPR) - Curitiba, PR - Brasil \\ Programa de Pós-Graduação em Engenharia Elétrica e Informática Industrial \\ \{daniloassis, joilson, ewille\}eutfpr.edu.br
}

\begin{abstract}
Vehicular Ad Hoc Networks (VANETs) are part of Intelligent Transportation Systems (ITS) and their main objective is to provide communication between vehicles. As self-organizable and self-manageable networks, with decentralized control, their performance is totally dependent on the route duration times. This study proposes an analysis of the route duration times in vehicular networks, considering three influential factors: speed, density and travel orientation. Simulation experiments corroborate that the route duration times increases in denser networks and when vehicles travel in the same orientation. In addition, the route duration times do not undergo significant changes depending on the speed of the vehicles when these speeds are limited by the environment (as in the case of the urban vehicle environment).
\end{abstract}

Resumo. As Redes Ad Hoc Veiculares (VANETs) fazem parte dos Sistemas Inteligentes de Transporte (ITS) e têm por objetivo prover a comunicação entre veículos. Por serem redes auto-organizáveis e auto gerenciáveis com controle descentralizado, seu desempenho é totalmente dependente do tempo de duração das rotas. Este trabalho propõe a análise do tempo de duração de rotas em redes veiculares considerando três fatores influentes: velocidade, densidade e sentido dos deslocamentos. Experimentos de simulação comprovam que o tempo de duração de rotas aumenta em redes mais densas e quando os veículos trafegam no mesmo sentido. Além disso, o tempo de duração de rotas não sofre modificações significativas em função da velocidade dos veículos quando essas são limitadas pelo ambiente (como no caso do ambiente veicular urbano).

\section{Introdução}

As redes $A d H o c$ veiculares (VANETs) são redes onde a comunicação é estabelecida entre veículos (carros, caminhões, ônibus, etc) [Alves Jr e Wille 2015]. Estas redes fazem parte dos Sistemas Inteligentes de Transporte (ITS) e devem ser capazes de prover segurança e entretenimento aos motoristas e passageiros [Li et al. 2016].

Os nós de tais redes se comunicam uns com os outros por meio de sinais de radiofrequência. Como o alcance dos sinais de rádio é limitado, cada nó só pode se comunicar diretamente com veículos que estiverem dentro do raio de transmissão de seus sinais. Contudo, pode existir a necessidade de um veículo transmitir informações para outros veículos que estão além do seu raio de transmissão [Almohammedi 2016]. Para isso, os veículos devem cooperar entre si, agindo como roteadores (descobrindo rotas e repassando as informações da origem até o destino) [Alves Jr e Wille 2016]. 
O envio/recebimento das informações ocorre somente quando um veículo de origem possui uma rota para um veículo de destino. Como as redes veiculares são redes auto gerenciáveis, auto-organizáveis, com controle descentralizado, o seu desempenho é totalmente dependente da existência de rotas e do tempo em que as mesmas permanecem estabelecidas (enquanto uma rota permanecer estabelecida, a origem e o destino são capazes de enviar e receber dados) [Alves Jr e Wille 2016].

Nas VANETs, os principais desafios da comunicação estão relacionados a problemas de conectividade entre os veículos, devido ao baixo tempo de duração das rotas [Liu et al. 2018]. Conhecer e avaliar o tempo de duração das rotas e os fatores que mais influenciam neste tempo, pode ajudar no desenvolvimento de protocolos e aplicações mais adequados para maximizar a conectividade da rede, assim como contribuir para o desenvolvimento de uma infraestrutura de comunicação veicular mais eficiente.

A maioria das análises em VANETs sobre o tempo de duração das rotas e da conectividade são realizadas por meio de estudos numéricos, [Raw et al. 2014], [Muhammed Ajeer et al. 2011], [Nazar e Alsabbagh 2016]. Tais estudos levam em consideração a velocidade, a densidade, o sentido de deslocamentos (os fatores influentes), mas não consideram as condições da estrutura viária (ruas, quadras, cruzamentos e semáforos) e do fluxo de veículos (congestionamentos). Sendo assim, alguns de seus resultados podem não representar fielmente a realidade dos movimentos veiculares.

Este trabalho propõe a análise do tempo de duração de rotas, da conectividade geral e de métricas de desempenho com o objetivo de evidenciar qual é o fator mais importante a ser considerado dado o ambiente onde um sistema irá atuar. A lógica de análise se dá por comparação entre situações diferentes. A primeira investigação considera um conjunto de dois cenários com características opostas em mobilidade. De um lado um sistema irrestrito em termos de movimento dos nós, de outro lado o movimento dos nós é condicionado pela disposição de ruas em um ambiente urbano e homogêneo. A segunda investigação considera um cenário cuja e única finalidade é avaliar o impacto da direção do movimento dos nós no desempenho da rede.

O restante deste trabalho está organizado da seguinte forma: a Seção 2 exibe os trabalhos relacionados; a Seção 3 apresenta a análise de desempenho de uma rede veicular; a Seção 4 mostra o resultado das simulações. Finalmente, a Seção 5 apresenta a conclusão.

\section{Trabalhos relacionados}

Os autores em [Liu et al. 2018] desenvolveram o RAODV, um protocolo de roteamento que leva em consideração a velocidade dos nós para selecionar a rota mais estável em MANETs (aquela que apresenta a menor velocidade média). Resultados de simulações mostraram que o protocolo é capaz eleger rotas estáveis, diminuindo a sobrecarga da rede e aumentando a taxa de entrega.

Os autores em [Aliesawi et al. 2018] apresentaram um protocolo de roteamento, o U-AODV. O objetivo deste protocolo é minimizar as quebras de link que ocorrem na rede. Para isso, ele considera fatores como a velocidade e o sentido dos deslocamentos dos veículos para selecionar o próximo salto durante a fase de descoberta de rotas. Por diminuir as quebras de links, o U-AODV se mostrou eficaz quando comparado ao protocolo 
AODV, reduzindo a sobrecarga da rede e aumentando a taxa de entrega dos pacotes.

O estudo em [Hussain et al. 2019] considerou fatores como a velocidade, o raio de transmissão e o comprimento da via para propor uma aplicação capaz de diminuir o congestionamento de veículos em uma praça de pedágio. Os veículos que utilizam essa aplicação, e já estão na praça de pedágio, informam os outros veículos que ainda estão a caminho para que possam reduzir a velocidade, e assim minimizar a formação de filas. Os resultados das simulações confirmaram que o sistema é capaz de diminuir o congestionamento.

Os autores em [Umer e Afzal 2018] propuseram um modelo de conectividade formado por um anel duplo de comunicação. O modelo leva em consideração a quantidade e a velocidade dos diferentes tipos de veículos (ônibus e automóveis) presentes na via. Este modelo é formado por um anel primário e um secundário. Os veículos que se deslocam rapidamente (automóveis) formam o anel primário. Já os veículos que se deslocam com baixa velocidade (ônibus), formam o anel secundário. Quando ocorre a quebra de rotas em função da velocidade dos veículos no anel primário, o anel secundário pode ser utilizado para a formação de novas rotas (a probabilidade de quebra nele em função da velocidade é menor). Esse estudo mostrou-se eficaz no aumento da conectividade da rede, diminuindo o atraso da entrega dos pacotes de dados.

Os autores em [Manel e Lamia 2017] apresentaram o protocolo de roteamento Speed based Ad Hoc On Demand Vector link Routing Protocol (SODV). O SODV leva consideração a média geométrica das velocidades instantâneas dos veículos para formar a rota mais estável (a que possui a média geométrica mais próxima da velocidade instantânea da origem). A média geométrica das velocidades foi escolhida por retratar melhor a realidade do que a média aritmética. Experimentos de simulação comprovam que o protocolo SODV apresentou um atraso menor em relação ao protocolo de roteamento AODV.

Desta forma, nota-se que existem trabalhos que consideram problemas relacionados a conectividade da rede e a quebra de links/rotas, além de utilizarem os fatores influentes para diversos fins. Entretanto, não observou-se a utilização de tais fatores para análises sobre o tempo de duração de rotas ou para determinar a influência dos mesmos na conectividade das VANETs.

\section{Análise de Desempenho de uma Rede Veicular}

Nesta seção são apresentadas métricas que permitem avaliar o desempenho de uma rede veicular. Além disso, uma métrica padronizada única (baseada no tempo de duração de rotas) é proposta para análise dos casos analisados neste trabalho.

\subsection{Métricas para avaliação de desempenho}

As seguintes métricas são consideradas:

- Taxa de Duração de Rota (TDR): percentagem de tempo onde existe conexão durante o tempo de simulação. A Equação 1 define formalmente o TDR:

$$
T D R=\left(\frac{x}{t}\right) * 100
$$

onde $x$ é a média dos tempos de duração de todas as rotas formadas e $t$ é o tempo total de simulação. 
- Índice de Quebra de Rota (IQR): é a quantidade de vezes que ocorreu a quebra de uma rota estabelecida.

- Taxa de Entrega de Pacotes (TEP): É a proporção do número total de pacotes recebidos no destino $\left(N_{r}\right)$ em relação ao número total de pacotes enviados pela origem $\left(N_{g}\right)$, i.e., $T E P=N_{r} / N_{g}$.

- Sobrecarga de Roteamento (SR): é a proporção do número total de pacotes de dados recebidos no destino $\left(N_{r e}\right)$, em relação ao número total de pacotes de roteamento enviados pela origem $\left(N_{d r}\right)$, i.e., $S R=N_{r e} / N_{d r}$.

- Atraso Médio de Pacote (AMP): é o atraso médio sofrido pelos pacotes de dados para propagar da origem ao destino. O atraso inclui o tempo total de transmissão, ou seja, tempo de propagação, tempo de espera (fila), tempo de estabelecimento da rota, etc. A Equação 2 define formalmente o AMP:

$$
A M P=\frac{1}{N_{p}} \sum_{i=1}^{N_{p}}(t r-t e),
$$

onde $t r$ é o instante de tempo exato que o destino recebe o pacote, te é o instante de tempo exato que a origem envia o pacote e $N_{p}$ é o número de pacotes enviados.

\subsection{Conectividade geral da rede}

Conforme definido pela Equação 3, o tempo de duração de rotas é o intervalo de tempo em que um veículo de origem e um de destino permanecem conectados com no mínimo dois saltos (caso não exista dois saltos, forma-se uma conexão direta (link)).

$$
\Delta T=t_{2}-t_{1}, \forall r \geq 2
$$

onde $t_{1}$ é o tempo exato em que uma certa rota foi estabelecida, $t_{2}$ é o tempo exato em que uma certa rota foi interrompida e $r$ o número de saltos.

Conforme definido pela Equação 4, a conectividade geral da rede $(C)$ é a média dos tempos de duração de todas as rotas estabelecidas na rede, durante um determinado intervalo de tempo.

$$
C=\frac{1}{n} \sum_{i=1}^{n} \Delta T^{(i)}
$$

onde $\Delta T^{(i)}$ é o tempo de duração da rota $i$ e $n$ é o número de rotas estabelecidas.

\subsection{Cenários de simulação}

Nesse trabalho são utilizados três cenários de simulação. A lógica de análise se dá por comparação entre duas situações diferentes. Desta forma os resultados obtidos a partir dos Cenários 1 e 2 são comparados entre si para evidenciar o efeito da mobilidade dos nós no desempenho da rede. De um lado tem-se um sistema irrestrito em termos de movimento dos nós (Cenário 1), de outro lado o movimento dos nós é condicionado pela disposição de ruas em um ambiente urbano e homogêneo (Cenário 2). A razão para 
tal escolha reside no fato de que casos intermediários (de interesse prático) encontramse posicionados em algum ponto entre esses extremos. O (Cenário 3) tem como único objetivo avaliar o impacto da direção do movimento dos nós no desempenho da rede. Para as análises foi utilizando o simulador de rede Network Simulator (NS-2) [Greis 2019], o simulador de mobilidade veicular VanetMobiSim [Härri et al. 2006] e o simulador de mobilidade aleatória Setdest [Sarkar et al. 2018].

\subsubsection{Cenário 1}

Conforme ilustrado na Figura 1(a), o Cenário 1 é uma área quadrangular de $1000 \mathrm{~m}$ por $1000 \mathrm{~m}$ sem ruas ou quadras. Neste cenário, os veículos se deslocam de acordo com o modelo de mobilidade Random Way Point (RWP). No RWP, cada nó permanece parado por um período de tempo em um intervalo definido. Após, os nós escolhem aleatoriamente um dos caminhos possíveis a partir de seu ponto inicial, em uma velocidade distribuída entre os parâmetros MIN e MAX. Ao chegar ao destino, o nó permanece parado um determinado tempo e reinicia o processo. Para esse cenário, o tempo de parada dos veículos é de 1 segundo [Purnomo et al. 2018]. Este cenário proporciona uma mobilidade livre para os veículos sem obstáculos, estradas ou cruzamentos. O contato entre os veículos é de curta duração devido a inexistência de restrições viárias [Mahajan et al. 2010].

\subsubsection{Cenário 2}

Conforme ilustrado pela Figura 1(b), o Cenário 2 é uma área quadrangular de $1000 \mathrm{~m}$ por $1000 \mathrm{~m}$ com quadras de $100 \mathrm{~m}$ por $100 \mathrm{~m}$ [Alam et al. 2009]. Quadras de $100 \mathrm{~m} \times 100 \mathrm{~m}$ foram escolhidas por ser esse um valor padrão na maioria das cidades. Este cenário corresponde a uma grade de Manhattan com ruas horizontais, verticais e interseções que modelam um ambiente real e urbano [Mir e Filali 2014, Spaho et al. 2013, Akkari Sallum et al. 2018]. Os veículos se deslocam de acordo com modelo de mobilidade implementado no software VanetMobiSim, que considera um sistema com pista dupla, aceleração, desaceleração e ultrapassagens sem colisões. Alguns semáforos dispostos aleatoriamente estão presentes. A velocidade de um veículo é condicionada pela velocidade do veículo que está à sua frente.

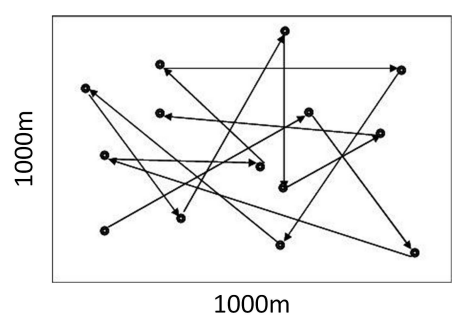

(a) Cenário 1

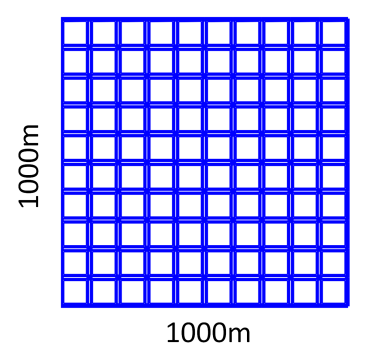

(b) Cenário 2

Figura 1. Cenários 1 e 2. 


\subsubsection{Cenário 3}

Conforme ilustrado na Figura 2(a), no Cenário 3 existem duas áreas retangulares (com 30 veículos em cada área) de 2000m x 1000m, em que os veículos se deslocam de acordo com modelo de mobilidade do VanetMobiSim. $\mathrm{Na}$ área $\mathrm{B}$, os veículos se deslocam obedecendo o sentido horário. $\mathrm{Na}$ área $\mathrm{A}$, os veículos podem se deslocar em sentido horário ou anti-horário. Desta forma, na região central, duas situações podem ocorrer: o estabelecimento de rotas com veículos avançando no mesmo sentido ou em sentidos opostos.

A Figura 2(b) (recorte da área central da Figura 2(a)) mostra que foram inseridos 6 pontos fixos (road-side units) que atuam como origem e destino de forma cruzada. Entende-se por forma cruzada que: o ponto fixo 0 se comunica com o ponto 4 e o ponto 4 com o ponto 2 . O ponto fixo 3 se comunica com ponto 1 e o ponto 1 com o ponto 5 . Esta forma de comunicação foi utilizada para garantir que as rotas possam ser estabelecidas entre veículos nas áreas A e B.

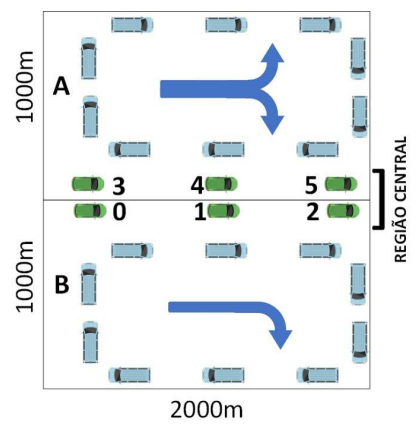

(a) Cenário 3

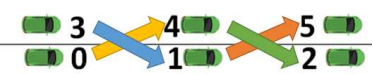

(b) A forma de comunicação cruzada

Figura 2. Cenário 3.

\subsubsection{Configuração das simulações}

O padrão de tráfego consiste de conexões com taxa de bits constante (CBR - com 4 mensagens/s), considerando o protocolo de transporte UDP e o protocolo de roteamento AODV [Akkari Sallum et al. 2018]. Um protocolo de roteamento clássico é suficiente para os objetivos deste trabalho. O modelo de propagação de rádio utilizado é o Two Rayground, enquanto a camada MAC está de acordo com a especificação IEEE 802.11p [Jiang e Delgrossi 2008]. São consideradas 5 conexões simultâneas, onde origem e destino são escolhidos ao acaso ao inicio e permanecem desta forma até o fim da simulação. As simulações são realizadas por 600 segundos para os Cenários 1 e 2 e por 2000 segundos para o Cenário 3. Todos os resultados apresentados são as médias de 35 simulações com o mesmo modelo de tráfego, mas com diferentes cenários de mobilidade. Para estas simulações o intervalo de confiança considerado é de $95 \%$.

\section{Resultados das simulações}

Nesta seção são apresentados os resultados das simulações de todos os modelos discutidos. Os gráficos apresentam no eixo "x" as variações dos fatores influentes (den- 
sidade, velocidade e sentido dos deslocamentos) e no eixo "y" os valores obtidos para as métricas de desempenho.

Inicialmente observa-se que, devido ao fato do VanetMobiSim representar mais fidedignamente o comportamento dos veículos (parada em semáforos e cruzamentos, frenagem para evitar colisão, aceleração e desaceleração), as velocidades médias (VLM) dos veículos no Cenário 2 ficam muito próximas, independentemente das velocidades máximas $(10,30$ e $60 \mathrm{~km} / \mathrm{h}$ ) que elas podem atingir (Figura 3). Isso sugere que o impacto da velocidade em ambientes reais é mitigado. Já o Cenário 1 corresponde a uma situação oposta de simulação onde os veículos estão livres em sua movimentação.

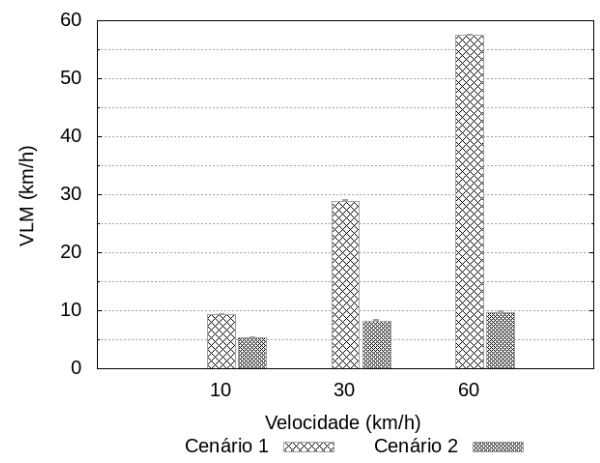

Figura 3. Velocidade média para o Modelo de simulação 1 e 2.

Outro fator que deve ser considerado ao se analisar os cenários, diz respeito à capacidade dos veículos estabelecerem conexões. Nos Cenários 1 e 2, um reduzido número de veículos (nós) ou raio de transmissão, diminui substancialmente a probabilidade de conexão na rede (ver o reduzido desenvolvimento analítico no Apêndice A) com efeitos negativos em todas as métricas.

\subsection{Modelo de simulação 1: análise da variação da velocidade}

Nesse caso os veículos possuem velocidades que variam entre $10 \mathrm{~km} / \mathrm{h}$ e $60 \mathrm{~km} / \mathrm{h}$, o raio de transmissão é de $250 \mathrm{~m}$ e o número de veículos varia de 10 à 100 . O objetivo é analisar a influência da velocidade no tempo de duração das rotas. Para realizar estas análises foram utilizados os Cenários 1 e 2 considerando-se 10 veículos (a), 50 veículos (b) e 100 veículos (c).

A Figura 4(a) apresenta o TDR em função da velocidade para o Cenário 1 e para o Cenário 2. Nota-se no Cenário $1(R W P)$ que a medida que a velocidade aumenta, o TDR diminui (como é esperado). Entretanto, para o Cenário 2 (VanetMobiSim), o TDR não diminui necessariamente pois os veículos dificilmente alcançam a velocidade máxima.

A Figura 4(b) apresenta o IQR versus a variação da velocidade. Como pode-se observar no Cenário 1, o IQR aumenta à medida que a velocidade dos veículos aumenta. Esse comportamento é observado em função do afastamento dos veículos além do raio de alcance dos sinais de radiofrequência uns dos outros, interrompendo a comunicação estabelecida. Entretanto, para o Cenário 2, como as velocidades médias desenvolvidas pelos veículos não apresentam grandes variações, as rotas não se interrompem com facilidade, pois os veículos permanecem por mais tempo dentro do raio de alcance uns dos outros. Assim, o IQR para um cenário mais realista não aumenta necessariamente conforme a elevação da velocidade. 


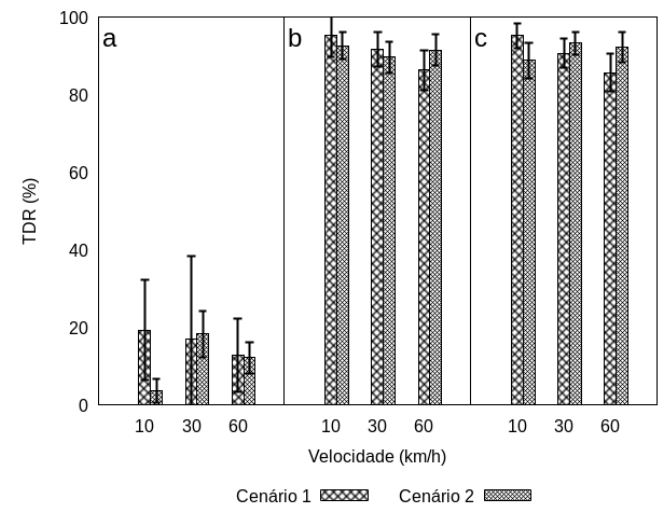

(a) TDR.

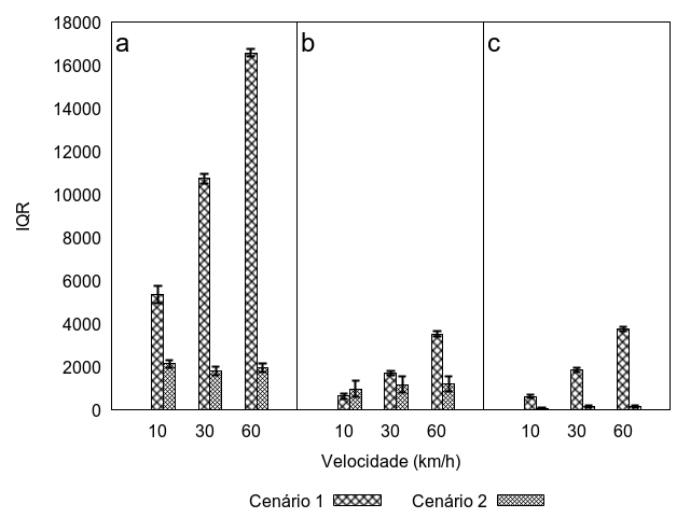

(b) IQR.

Figura 4. TDR e IQR para o modelo de simulação 1 e 2.

Observa-se na Figura 5(a) que, para o Cenário 1, o SR aumenta à medida que a velocidade dos veículos aumenta. Esse fato é observado em função das constantes quebras de rotas que ocorrem pela variação da velocidade. As quebras de rotas desencadeiam novos processos de descoberta, gerando mais pacotes de roteamento do que pacotes de dados na rede. Contudo, no cenário mais realista, o SR varia pouco à medida que a velocidade dos veículos aumenta (a exceção do caso onde a probabilidade de conexão é baixa).

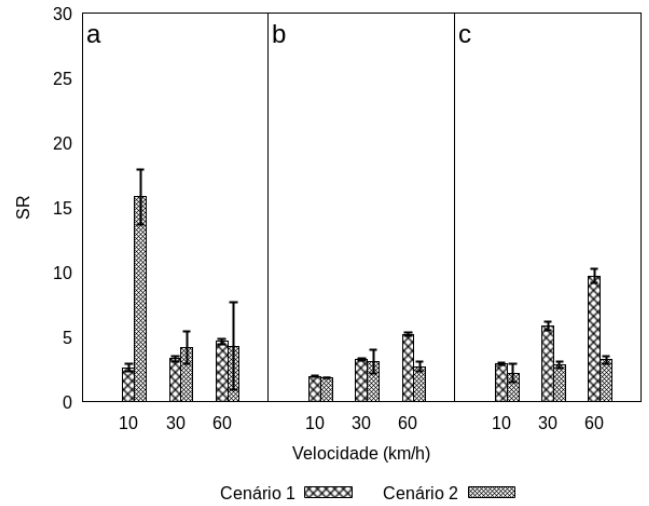

(a) SR.

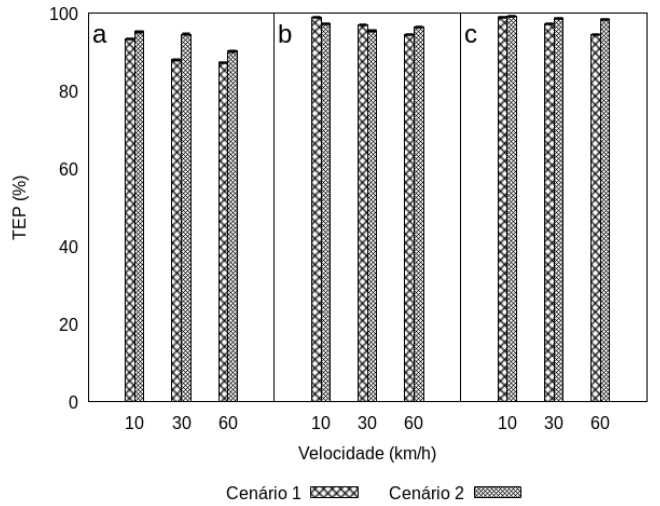

(b) TEP.

Figura 5. SR e TEP para o modelo de simulação 1 e 2.

Como pode-se observar na Figura 5(b), o TEP diminui ligeiramente conforme a velocidade dos veículos aumenta para o Cenário 1. Esse comportamento é observado em função do aumento das quebras de rotas. Entretanto, para o Cenário 2, o TEP não diminui necessariamente com o aumento da velocidade. Isso ocorre devido à baixa mobilidade veicular (velocidades médias próximas) acarretando em menos quebras de rotas.

A Figura 6 mostra o AMP em relação a velocidade de deslocamentos dos veículos para os Cenários 1 e 2. É possível observar que o AMP, no Cenário 1, apresenta valores maiores em relação ao Cenário 2. Isso acontece em função do aumento do número de quebras das rotas estabelecidas devido a alta mobilidade veicular, sendo necessário um tempo extra para que novas descobertas de rotas sejam concluídas [Liu et al. 2013]. 


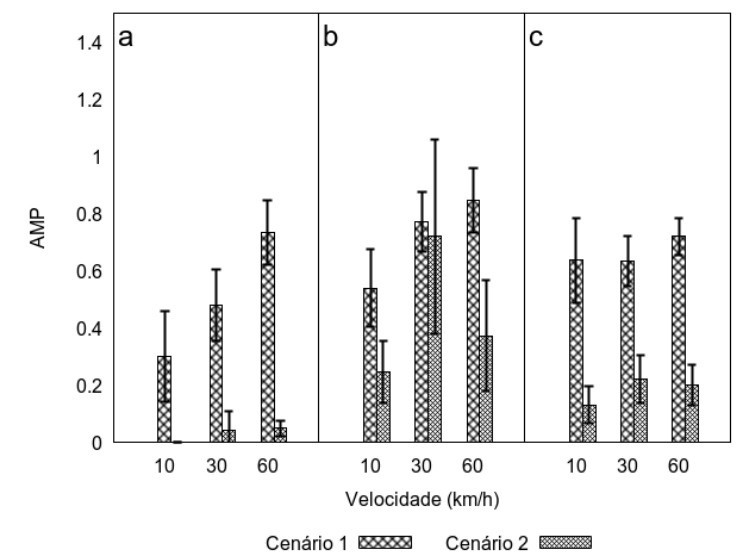

Figura 6. AMP para o Modelo de simulação 1 e 2.

\subsection{Modelo de simulação 2: análise da variação da densidade}

Quando as métricas de desempenho da rede são observadas, sob o ponto de vista da variação da densidade veicular (dado uma velocidade fixa), é possível observar que o TDR (Figura 4(a)) aumenta ou permanece estável à medida que número de veículos aumenta em ambos os cenários. Este fato é observado pois quanto mais densa a rede, maiores são as chances de rotas serem formadas (conforme apresentado no Apêndice A a probabilidade de conexão aumenta com a elevação do número de veículos).

Como pode-se notar na Figura 4(b), o IQR diminui ou permanece estável à medida que o número de veículos aumenta. Isso ocorre devido ao fato das rotas não se romperem com facilidade conforme o número de veículos aumenta.

A Figura 5(a) mostra que o SR diminui ou permanece estável a medida que o número de nós aumenta na rede. Isso ocorre em função da diminuição das quebras de rotas na rede em razão da alta densidade veicular. Como pode-se observar na Figura 5(b), o TEP aumenta a medida que o número de nós aumenta na rede. Isso ocorre em função da diminuição das interrupções das rotas estabelecidas na rede em razão da alta densidade veicular.

Entretanto, o atraso médio dos pacotes (Figura 6), para o Cenário 1, não diminui necessariamente à medida que o número de veículos aumenta. Esse fato é observado pois quando mais densa é a área mais pacotes de roteamento são encaminhados pela rede causando um maior atraso na entrega dos pacotes. Além disso, quando um veículo está transmitindo pacotes de dados e recebe pacotes de roteamento, a transmissão é interrompida, os pacotes de dados são armazenados em fila até que o protocolo encaminhe os pacotes de roteamento recebidos.

\subsection{Modelo de simulação 3: análise da variação do sentido dos deslocamentos}

Nesse caso são considerados os deslocamentos dos veículos no mesmo sentido e em sentido oposto. A velocidade varia de 10 à $60 \mathrm{~km} / \mathrm{h}$, o número de veículos é 66 e o raio de transmissão varia de $150 \mathrm{~m}$ à $350 \mathrm{~m}$. O objetivo é analisar a influência do sentido dos deslocamentos dos veículos no tempo de duração das rotas. Para realizar estas análises foi utilizado o Cenário 3 considerando-se um raio de transmissão de $150 \mathrm{~m}$ (a), 250m (b) e $350 \mathrm{~m}(\mathrm{c})$. 
A Figura 7(a) mostra o TDR em função do sentido dos deslocamentos versus a velocidade, para o Cenário 3, com raio de transmissão variando entre 150 à 350m. Notase que os veículos ao se deslocarem em Sentido Oposto (a partir de um raio de $250 \mathrm{~m}$ ), o TDR diminui ligeiramente à medida que a velocidade aumenta. Neste caso de deslocamento, os veículos saem com maior facilidade do raio de transmissão dos outros veículos e assim as rotas se rompem com maior facilidade, diminuindo o TDR. Porém, quando o tráfego ocorre no Mesmo Sentido, os veículos permanecem mais tempo dentro do raio de transmissão uns dos outros, e assim possibilita que as rotas permaneçam conectadas pelo maior tempo possível. Entretanto, com o raio de 150m, o TDR é muito baixo pois o contato entre os veículos é reduzido durante toda a simulação e a probabilidade de conexão (Apêndice A) é menor em relação aos outros raios de transmissão.

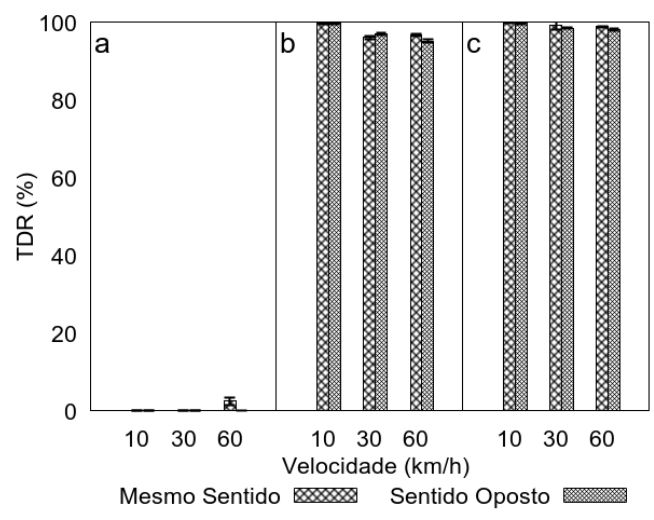

(a) TDR.

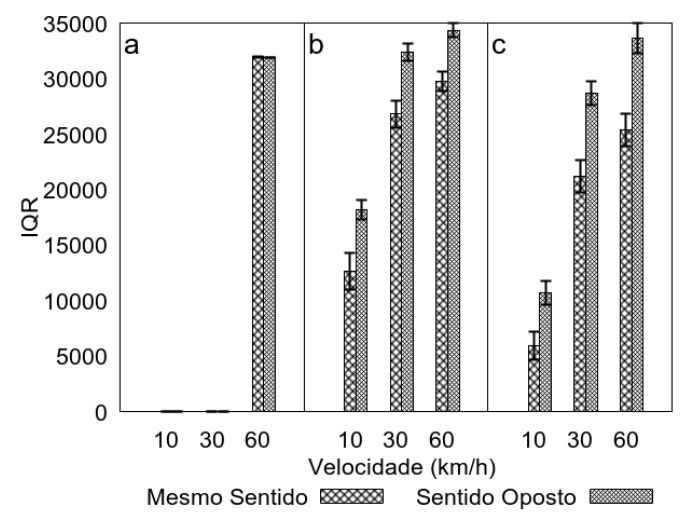

(b) IQR.

Figura 7. TDR e IQR para o modelo de simulação 3.

É possível notar que, na Figura 7(b), o IQR aumenta, para ambos os sentidos, à medida que a velocidade dos veículos aumenta. No Sentido Oposto, os veículos se afastam do raio de transmissão uns dos outros com maior facilidade. Esse afastamento, provoca diversas desconexões, causando o aumento do IQR. Entretanto, no Mesmo Sentido, embora as rotas sejam interrompidas, são rapidamente recuperadas pois a chance de um veículo entrar no raio de transmissão do outro é maior, em função do deslocamento dos veículos ocorrer sempre no Mesmo Sentido na região central do cenário.

Na Figura 8(a), observa-se que o SR aumenta com a velocidade. Neste caso, pode-se observar que devido às constantes quebras de rotas, o SR é maior no caso do Sentido Oposto. A Figura 8(b) mostra o TEP e é possível observar que o TEP diminui com o aumento da velocidade. Novamente encontra-se um melhor desempenho quando os veículos avançam no Mesmo Sentido.

Observa-se que as constantes quebras de rotas fazem com que o atraso aumente em função da velocidade (Figura 9) (quando ocorre a quebra de rotas, os pacotes de dados são enfileirados até que a rota seja novamente reestabelecida e com isso, o AMP aumenta). Veículos avançando em Sentido Oposto também elevam o AMP.

\subsection{Fatores com maior influência na conectividade geral da rede}

Esta seção, por meio da análise dos resultados fornecidos pela Equação 4, aplicado sobre todos os resultados dos cenários de cada Modelo de Simulação, tem o objetivo de 


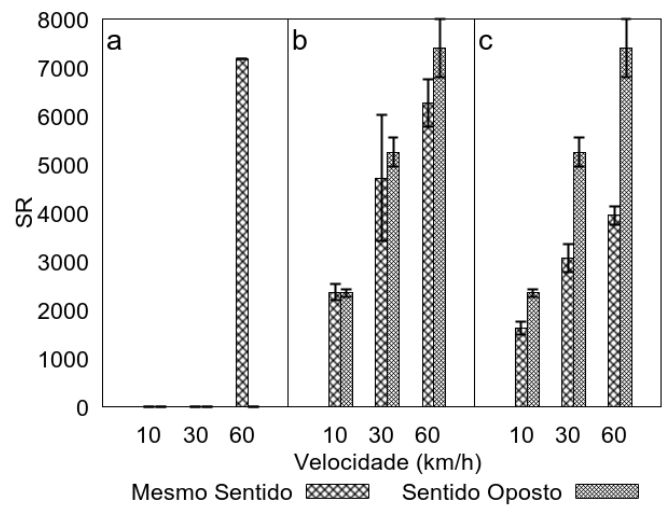

(a) SR.

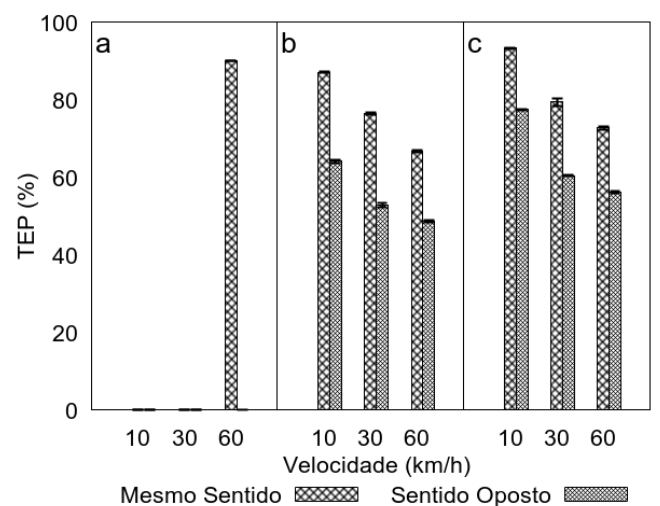

(b) TEP.

Figura 8. SR e TEP para o modelo de simulação 3.

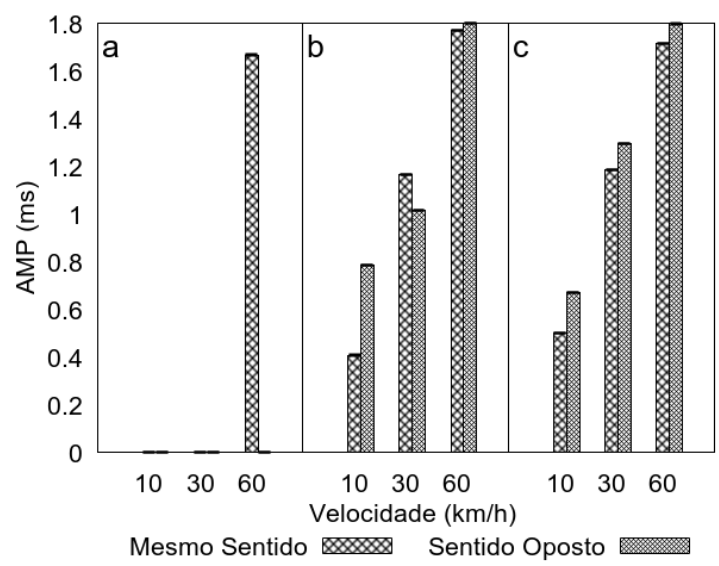

Figura 9. AMP para Modelo de simulação 3.

mostrar quais dos fatores (velocidade, densidade e sentido dos deslocamentos) possui a maior influência na conectividade geral da rede.

Conforme pode-se observar na Tabela 1 para o Cenário 1 e o Cenário 2, o fator que possui a maior influência na conectividade da rede (torna a conectividade maior) é a densidade, seguido pela velocidade. Esse comportamento é devido ao fato de a densidade impactar a probabilidade de conexão.

Tabela 1. Conectividade Geral da Rede: Velocidade e Densidade.

\begin{tabular}{|c|c|c|}
\hline Fatores & $\begin{array}{c}\text { Cenário 1 } \\
\text { Média (C) }\end{array}$ & $\begin{array}{c}\text { Cenário 2 } \\
\text { Média (C) }\end{array}$ \\
\hline Velocidade & 388,75 & 524,65 \\
\hline Densidade & 596,48 & 395,63 \\
\hline
\end{tabular}

Além disso, a Tabela 2 mostra que, no Cenário 3, o fator sentido de deslocamento possui influência na conectividade geral da rede. Observa-se tal comportamento pois o tráfego no mesmo sentido favorece a permanência dos veículos dentro do raio de alcance uns dos outros e contribui para o aumento da conectividade da rede. 
Tabela 2. Conectividade Geral da Rede: Sentido dos deslocamentos.

\begin{tabular}{|c|l|}
\hline Sentido & $\begin{array}{l}\text { Cenário 3 } \\
\text { Média (C) }\end{array}$ \\
\hline Mesmo Sentido & 1318,80 \\
\hline Sentido Oposto & 1307,55 \\
\hline
\end{tabular}

\section{Conclusão}

Este trabalho propôs a análise do impacto do tempo de duração de rotas e de métricas que impactam no desempenho das redes veiculares, considerando três fatores influentes, os quais são: velocidade, densidade e sentido do deslocamento dos veículos.

Experimentos de simulação mostraram que o TDR aumenta conforme a rede fica mais densa e quando os veículos se deslocam no mesmo sentido, minimizando as interrupções de rotas estabelecidas, reduzindo a sobrecarga na rede e contribuindo para o aumento da taxa de entrega dos pacotes. Entretanto, contrariando a lógica comum, o TDR não diminui com o aumento da velocidade em um cenário mais realista ( Cenário 2). Isso ocorre pelas velocidades médias dos veículos serem muito próximas devido as características da mobilidade (parada em semáforos e cruzamentos, frenagem para evitar colisão, aceleração e desaceleração).

Já no Cenário 1, o TDR diminui com o aumento da velocidade em função das constantes quebras de rotas que ocorrem pela alta mobilidade dos veículos, degradando o desempenho da rede (aumentando a sobrecarga de roteamento, diminuindo o TEP e aumentando o atraso na entrega dos pacotes). Além disso, as simulações mostraram que o TDR também aumenta ligeiramente ou permanece estável quando os veículos se deslocam no Mesmo Sentido. Neste caso, os veículos permanecem mais tempo dentro do raio de alcance dos sinais uns dos outros. Com isso, mesmo que ocorram as quebras de rotas, elas são rapidamente recuperadas. Entretanto, quando os veículos se deslocam em sentidos opostos, o TDR diminui em razão da elevação do número de rotas quebradas.

As simulações mostraram também, baseado-se na Equação 4, que o fator que tem maior influência na conectividade, no primeiro cenário é a velocidade veicular e no segundo é a densidade. Além disso, os experimentos de simulação envolvendo o Cenário 3 mostraram que o deslocamento relativo entre os veículos é fator influente na conectividade geral da rede, sendo que o deslocamento no Mesmo Sentido tem impacto positivo em todas as métricas.

Como proposta de trabalhos futuros pretende-se propor um novo protocolo de roteamento para redes veiculares que considera em sua função, o custo dos fatores que efetivamente impactam no desempenho do sistema conforme evidenciado neste trabalho.

\section{A. Probabilidade de conexão em uma rede sem fio}

Seja um conjunto de $n$ nós, cada qual com um raio de transmissão $r_{0}(m)$ distribuídos conforme um processo pontual homogêneo de Poisson em uma área $A\left(m^{2}\right)$. Segundo Bettstteter [Bettstetter 2002], a probabilidade $\left(P_{i}\right)$ de que um nó não tenha vizinhos (i.e., esteja isolado) é dado por: $P_{i}=e^{-\pi \rho r_{0}^{2}}$, onde $\rho=n / A$ é a densidade dos nós. Caso os nós estejam distribuídos sobre um eixo unidimensional de comprimento $x_{\max }$ $(m)$, então a probabilidade $P_{i}$ passa ser dada por: $P_{i}=e^{-2 \rho r_{0}}$, onde $\rho=n / x_{\text {max }}$ é a densidade dos nós. Assim, a probabilidade de conexão da rede (i.e., a probabilidade de que 
nenhum dos $n$ nós esteja isolado), assumindo eventos independentes, é: $P_{c}=\left(1-P_{i}\right)^{n}$. Resultados para $P_{c}$ são apresentados na Tabela 3 , onde $A=10^{6} \mathrm{~m}^{2}$ e $r_{0}=250 \mathrm{~m}$ (Cenário 1) e $x_{\max }=2000 \mathrm{~m}$ e $n=24$ nós (Cenário 3). Em função das limitações físicas impostas pelo Cenário 2, pode-se imaginar valores de probabilidade um pouco menores que aqueles do Cenário 3.

Tabela 3. Probabilidade de conexão nos Cenários 1 e 3.

\begin{tabular}{|l|c|c|c|}
\hline \multicolumn{4}{|c|}{ Cenário 1 } \\
\hline Veículos & $\mathbf{1 0}$ & $\mathbf{5 0}$ & $\mathbf{1 0 0}$ \\
\hline$P_{c}$ & $22,0 \%$ & $99,7 \%$ & $100 \%$ \\
\hline \multicolumn{4}{|c|}{ Cenário 3 } \\
\hline Raio (m) & $\mathbf{1 5 0}$ & $\mathbf{2 5 0}$ & $\mathbf{3 5 0}$ \\
\hline$P_{c}$ & $51,4 \%$ & $94,2 \%$ & $99.5 \%$ \\
\hline
\end{tabular}

Embora as equações apresentadas tenham sido obtidas para redes fixas, segundo Bettstteter, elas podem ser usadas para redes móveis desde que os efeitos de borda (nos cenários de simulação) possam ser minimizados.

\section{Referências}

[Akkari Sallum et al. 2018] Akkari Sallum, E. E., dos Santos, G., Alves, M., e Santos, M. M. (2018). Performance analysis and comparison of the DSDV, AODV and OLSRrouting protocols under VANETs. In 2018 16th International Conference on Intelligent Transportation Systems Telecommunications (ITST).

[Alam et al. 2009] Alam, M., Sher, M., e Husain, A. (2009). Integrated mobility model (IMM) for vanets simulation and its impact. In 2009 International Conference on Emerging Technologies.

[Aliesawi et al. 2018] Aliesawi, Salah e Mubarek, F., Ali Alheeti, K. M., e M. Alfahad, N. (2018). Urban-AODV: An improved AODV protocol for vehicular Ad-Hoc networks in urban environment. Int. Journal of Engineering e Technology(UAE), 7.

[Almohammedi 2016] Almohammedi, Akram e Noordin, N. e. S. S. (2016). Evaluating the impact of transmission range on the performance of VANET. Int. Journal of Electrical e Computer Engineering (IJECE).

[Alves Jr e Wille 2015] Alves Jr, J. e Wille, E. (2015). Improving VANETs connectivity with a totally Ad Hoc living mobile backbone. Journal of Computer Networks e Communications, 2015.

[Alves Jr e Wille 2016] Alves Jr, J. e Wille, E. (2016). P-AOMDV: An improved routing protocol for $\mathrm{V} 2 \mathrm{~V}$ communication based on public transport backbones. Transactions on Emerging Telecommunications Technologies.

[Bettstetter 2002] Bettstetter, C. (2002). On the minimum node degree and connectivity of a wireless multihop network. In Proceedings of the 3rd ACM International Symposium on Mobile Ad Hoc Networking and Computing, New York, NY, USA. ACM.

[Greis 2019] Greis, M. (2019). The Network Simulator - (NS-2).

[Härri et al. 2006] Härri, J., Filali, F., Bonnet, C., e Fiore, M. (2006). VanetMobiSim: Generating realistic mobility patterns for vanets. In Proceedings of the 3rd International Workshop on Vehicular Ad Hoc Networks, New York, NY, USA. ACM. 
[Hussain et al. 2019] Hussain, S., Wu, D., Memon, S., e Khuda Bux, N. (2019). Vehicular Ad Hoc network (VANET) connectivity analysis of a highway toll plaza. Data.

[Jiang e Delgrossi 2008] Jiang, D. e Delgrossi, L. (2008). IEEE 802.11p: Towards an international standard for wireless access in vehicular environments. In VTC Spring, pgs 2036-2040. IEEE.

[Li et al. 2016] Li, C., Zhen, A., Sun, J., Zhang, M., e Hu, X. (2016). Analysis of connectivity probability in VANETs considering minimum safety distance. In 2016 8th International Conference on Wireless Communications Signal Processing (WCSP).

[Liu et al. 2013] Liu, S., Yang, Y., e Wang, W. (2013). Research of AODV routing protocol for ad hoc networks. AASRI Procedia, 5:21 - 31. 2013 AASRI Conference on Parallel and Distributed Computing and Systems.

[Liu et al. 2018] Liu, T., Xia, Z., Shi, S., e Gu, X. (2018). A modified AODV protocol based on nodes velocity. In Gu, X., Liu, G., e Li, B., editors, Machine Learning and Intelligent Communications, Cham. Springer International Publishing.

[Mahajan et al. 2010] Mahajan, A., Potnis, N., Gopalan, K., e Wang, A.-I. (2010). Urban mobility models for VANETs.

[Manel e Lamia 2017] Manel, K. e Lamia, C. (2017). SODV speed based Ad Hoc on demand vector link routing protocol: A routing protocol for VANET networks. In 2017 Sixth International Conference on Communications and Networking (ComNet).

[Mir e Filali 2014] Mir, Z. e Filali, F. (2014). LTE and IEEE 802.11p for vehicular networking: A performance evaluation. EURASIP Journal on Wireless Communications and Networking, 2014.

[Muhammed Ajeer et al. 2011] Muhammed Ajeer, V. K., Neelakantan, P. C., e Babu, A. V. (2011). Network connectivity of one-dimensional vehicular Ad Hoc network. In 2011 International Conference on Communications and Signal Processing.

[Nazar e Alsabbagh 2016] Nazar, R. e Alsabbagh, H. (2016). Analysis of path duration in VANETs using B-MFR forwarding method.

[Purnomo et al. 2018] Purnomo, A., Widyawan, Najib, W., Hartono, R., e Hartatik (2018). Effect of variation in active route timeout and my route timeout on the performance of AODV-ETX protocol in mobile adhoc network. IOP Conference Series: Materials Science and Engineering.

[Raw et al. 2014] Raw, R., Kumar Soni, S., Singh, N., e Kaiwartya, O. (2014). A probabilistic analysis of path duration using routing protocol in VANETs. Int. Journal of Vehicular Technology, 2014.

[Sarkar et al. 2018] Sarkar, D., Choudhury, S., e Majumder, A. (2018). Enhanced-antAODV for optimal route selection in mobile Ad-Hoc network. Journal of King Saud University - Computer e Information Sciences.

[Spaho et al. 2013] Spaho, E., Ikeda, M., Barolli, L., Xhafa, F., Kolici, V., e Iwashige, J. (2013). Performance evaluation of AODV Routing Protocol in VANETs considering multi-flows traffic. In 2013 Eighth International Conference on Broadband and Wireless Computing, Communication and Applications.

[Umer e Afzal 2018] Umer, T. e Afzal, M. K. (2018). A dual ring connectivity model for VANET under heterogeneous traffic flow. Wireless Personal Communications. 